\title{
In situ measurement of miR-205 in malignant melanoma tissue supports its role as a tumor suppressor microRNA
}

\author{
Jason A Hanna, Lewis Hahn, Seema Agarwal and David L Rimm
}

Oncogenic and tumor suppressing microRNAs (miRNAs) have emerged as key regulators of gene expression in many types of cancer including melanoma. We utilized quantitative in situ hybridization (qISH) to evaluate the tumor suppressing properties of miRNA, miR-205 in a population of human tumors. We hypothesize decreased miR-205 would be associated with more aggressive tumors. Multiplexing miR-205 qISH with immunofluorescent assessment of S100/GP100 allowed us to quantitatively evaluate miR-205 expression using the AQUA method of quantitative immunofluorescence. The specificity of the assay was validated using blocking oligos and transfected cell lines as controls. Outcomes were assessed on the Yale Melanoma Discovery Cohort consisting of 105 primary melanoma specimens and validated on an independent set of 206 primary melanomas (Yale Melanoma Validation Cohort). Measurement of melanoma cell miR-205 levels shows a significantly shorter melanoma-specific survival in patients with low expression. Multivariate analysis shows miR-205 levels are significantly independent of stage, age, gender, and Breslow depth. Low levels of melanoma cell miR205 expression as quantified by ISH show worse outcome, supporting the role of miR-205 as a tumor suppressor miRNA. The quantification of miR-205 in situ suggests potential for the use of miRNAs in future prognostic or predictive models. Laboratory Investigation (2012) 92, 1390-1397; doi:10.1038/labinvest.2012.119; published online 13 August 2012

KEYWORDS: AQUA; melanoma; microRNA; mir-205; miRNA in situ hybridization

MicroRNAs (miRNAs) are a class of short noncoding RNAs of 18-25 nucleotides, which regulate gene expression by promoting transcript degradation or inhibiting translation of target mRNAs. ${ }^{1}$ miRNAs can be expressed at levels as high as 50000 copies per cell and with over 1500 human miRNAs it has been estimated that they regulate $30-60 \%$ of all mRNAs. ${ }^{2-4}$ miRNAs have key roles in proliferation, apoptosis, and differentiation in normal physiology, and their improper regulation has been attributed to cancer with scores of papers indicating miRNAs as functioning oncogenes or tumor suppressors. ${ }^{5}$

miR-205 is one such miRNA often implicated in a tumor suppressing role where it is downregulated or lost in transformation. ${ }^{6-9}$ In melanoma specifically, it is downregulated in tumor samples compared with nevi and decreases cell proliferation when overexpressed in melanoma cell lines. ${ }^{6,9,10}$ miR-205 expression is currently used clinically by Rosetta Genomics to distinguish squamous cell carcinoma from adenocarcinoma of the lung, ${ }^{11}$ but its role as a tumor suppressor has not been evaluated in populations of primary melanoma. Here, we attempt to validate the tumor suppressor function by association with outcome in two independent cohorts.

Most miRNA measurement methods require total RNA extracts, which lack spatial information and are subject to assessment of tumor and stroma in unknown mixtures. Measurement of miRNA by in situ hybridization (ISH) allows for the visualization of miRNA expression within individual cells providing the means by which to determine expression level in malignant as well as stromal and normal cells. This method also allows large scale specimen analysis on tissue microarrays (TMAs) with the previously described advantages related to standardization. In this study we evaluate the levels of miR-205 in two melanoma cohorts by quantitative ISH (qISH).

\section{MATERIALS AND METHODS Patients and Tumor Samples}

The Yale Melanoma Discovery Cohort and TMA construction have been described previously. ${ }^{12}$ The Yale Melanoma Discovery Cohort consisted of 158 white patients who underwent resection of a primary invasive cutaneous 
melanoma at Yale-New Haven Hospital between 1959-1994 where the specimen was not exhausted and follow-up information existed The array also contained 301 metastases, lymph node metastases, or visceral metastases occurring in patients diagnosed with cutaneous melanoma and surgically removed at Yale-New Haven Hospital from 1959 to 1994 or 1995 to 2002. The Yale Validation Cohort consisted of 315 white patients with invasive cutaneous malignant melanoma with excisions from 1995 to 2005 at Yale-New Haven Hospital; the array also contained 19 metastatic melanoma control spots. Clinical data were comprehensively obtained for the primary melanomas after review of medical records, the archives of the Connecticut Tumor Registry, and the State of Connecticut Vital Records. This study was approved by the Yale Human Investigations Committee. One hundered percent inclusion of patients was not attained in all analyses because of attrition of sample, diagnostic tissue exhaustion, obscuring lymphocyte infiltration, technical failure, or missing clinical information. Clinicopathological characteristics of the cohorts are shown in Table 1.

\section{MiRNA ISH}

miRNA ISH was performed as previously described. ${ }^{13}$ In brief, formalin-fixed paraffin-embedded (FFPE) TMAs are first melted at $60^{\circ} \mathrm{C}$ for $10 \mathrm{~min}$ then deparaffinized in three exchanges of xylene, followed by rehydration in an ethanol gradient. Then slides are treated with $20 \mu \mathrm{g} / \mathrm{ml}$ proteinase $\mathrm{K}$ (Roche Diagnostics, Indianapolis, IN, USA) for $10 \mathrm{~min}$ at $37^{\circ} \mathrm{C}$, fixed with $4 \%$ formaldehyde (Thermo Scientific, Rockford, IL, USA) in PBS, rinsed twice in $0.13 \mathrm{M}$ 1-methylimidazole (Sigma-Aldrich, St Louis, MO, USA) and refixed with 1-ethyl-3-(3-dimethylamino propyl) carbodiimide (EDC, Thermo Scientific) as described previously. ${ }^{14}$ Next endogenous peroxidases are blocked in $1 \% \mathrm{H}_{2} \mathrm{O}_{2}$ for $30 \mathrm{~min}$, and slides are prehybridized at the hybridization temperature $\left(50{ }^{\circ} \mathrm{C}\right)$ in a hybridization oven (Advanced Cell Diagnostics, Hayward, CA, USA) for $30 \mathrm{~min}$ in hybridization buffer with $500 \mu \mathrm{g} / \mathrm{ml}$ yeast tRNA (Invitrogen, Carlsbad, CA, USA), 50\% formamide (American Bioanalytical, Natick, MA, USA), $5 \times$ SSC (American Bioanalytical), $50 \mu \mathrm{g} / \mathrm{ml}$ Heparin (SigmaAldrich), 0.1\% Tween-20 (Sigma-Aldrich), adjusted to $\mathrm{pH}$ 6. Slides are hybridized for $1 \mathrm{~h}$ with $200 \mathrm{nM}$ double Digoxigenin (DIG) LNA-modified probes(Exiqon, Copenhagen, Denmark) for miR-205 (sequence: 5'-CAGAC TCCGGTGGAATGAAGGA- $3^{\prime}$ ) and scrambled probe (sequence: $5^{\prime}$-GTGTAACACGTCTATACGCCCA- $3^{\prime}$ ), and $25 \mathrm{nM} 5^{\prime}$ DIG labeled U6 probe (sequence: 5'-CACGA ATTTGCGTGTCATCCTT- $3^{\prime}$ ). Slides are then stringently washed in $2 \times$ SSC (once at hybridization temperature, then twice at room temperature for $5 \mathrm{~min}$ each), blocked with 2\% BSA (Sigma-Aldrich) for $30 \mathrm{~min}$ and incubated with Anti-DIG-POD, Fab fragments from sheep (Roche Diagnostics) diluted 1:100, rabbit polyclonal anti-S100 (Z0311, Dako, Carpinteria, CA, USA) diluted 1:100 and
Table 1 Clinicopathological characteristics of melanoma cohorts

\begin{tabular}{lcr} 
Characteristic & $\begin{array}{l}\text { Discovery } \\
\text { cohort, } n=113\end{array}$ & $\begin{array}{l}\text { Validation } \\
\text { cohort, } n=\end{array}$ \\
\hline Breslow thickness, $m m$ & & 3.45 \\
Mean & 3.08 & 4.61 \\
s.d. & 2.25 &
\end{tabular}

Age at diagnosis, years

$\begin{array}{lll}\text { Mean } & 58.24 & 63.43 \\ \text { s.d. } & 14.43 & 16.80\end{array}$

Sex

$\begin{array}{lrr}\text { Male } & 58(50.5 \%) & 80(38.8 \%) \\ \text { Female } & 54(49.5 \%) & 126(61.2 \%)\end{array}$

Stage at diagnosis

Localized

$89(82.4 \%)$

$169(82 \%)$

Regional

$11(10.2 \%)$

$33(16 \%)$

Distant

$8(7.4 \%)$

$4(1.9 \%)$

Ulceration

Absent

$67(59.3 \%)$

$67(52.3 \%)$

Present

$46(40.7 \%)$

$61(47.7 \%)$

Microsatellites

Absent

$86(76.1 \%)$

$71(83.5 \%)$

Present

$27(23.9 \%)$

$14(16.5 \%)$

Received any therapy

$\begin{array}{llr}\text { No } & 90(81.1 \%) & 153(81.4 \%) \\ \text { Yes } & 21(18.9 \%) & 35(18.6 \%)\end{array}$

rabbit polyclonal anti-GP100 diluted 1:25 (ab27435, Abcam, Cambridge, MA, USA) for $1 \mathrm{~h}$ at room temperature. Slides are then washed twice with $0.1 \%$ Tween-20 PBS (PBS-T) and once with PBS for $5 \mathrm{~min}$ each. Then the miRNA signal is detected with the TSA Plus Cyanine 5 system (Perkin Elmer, Norwalk, CT, USA), the slides are washed with PBS-T and PBS as above, and the S100/GP100 is detected with Alexa 546-conjugated goat anti-rabbit secondary antibody (Molecular Probes, Eugene, OR, USA) diluted 1:100 in block for $1 \mathrm{~h}$. The slides are washed in PBS-T and PBS then mounted with Prolong mounting medium containing 4', 6-Diamidino-2-phenylindole (DAPI, Molecular Probes). Serial sections of control index arrays consisting of 20 nevi, 20 primary, and 20 metastatic melanoma specimens are simultaneously run to assess reproducibility. In addition, 
negative control scrambled probe and positive control U6 probes are run with each experiment. The miR-205 blocking oligo (sequence: $5^{\prime}$-TCCTTCATTCCACCGGAGTCTG- $3^{\prime}$ ) and mutated blocking oligo (sequence: $5^{\prime}$-TCCTACA TTCGACCGGTGTCTG- $3^{\prime}$ ) were preincubated at four-fold excess $(800 \mathrm{nM})$ with the DIG labeled miR-205 probe for $1 \mathrm{~h}$ at the hybridization temperature before hybridization on the TMA.

\section{Quantitative Immunofluorescence (qIF)}

The AQUA technology for qIF to measure biomolecules within subcellular compartments has been described previously. ${ }^{15}$ In brief, a series of monochromatic in and out of focus images are obtained for each histospot using the signal from the DAPI, S100/GP100-Alexa 546, and the Cy5 (miRNA/U6) channel by the PM-2000 microscope. A tumor 'mask' is created from the S100/GP100 signal to distinguish stromal from tumor area. This tumor mask is based on an intensity threshold set upon visual inspection of each histospot. AQUA scores of miR-205 are calculated by dividing the signal intensity (scored on a scale from 0 to 255 ) by the area of the tumor mask.

For statistical analysis, AQUA scores were averaged for individuals with multiple histospots on any array before analysis. miR-205 qISH was performed on two builds (redundant cores from different areas of same tumor specimens) of both the Discovery cohort and Validation cohort. The AQUA scores from the two cores were normalized and averaged for analysis. Averaged scores from the two cohorts were then median normalized to compare a single cut point on both cohorts. Any specimen containing less than $0.17 \mathrm{~mm}^{2}$ tumor area was excluded from analysis.

\section{Cell Culture}

Cell culture conditions have been previously described. ${ }^{15}$ Mel-501 cells were obtained from Dr Steven Rosenberg, Surgery Branch, National Cancer Institute (Bethesda, MD, USA) and A431 cells were purchased from ATCC. The A431 cells were transfected with $30 \mathrm{nM}$ miR-205 mirVana miRNA inhibitor (an antagomir or anti-sense RNA for miR-205) or negative control miRNA inhibitor (Ambion, Austin, TX, USA) and the Mel-501 cells were transfected with $30 \mathrm{nM}$ miR-205 mirVana miRNA mimic or mimic negative control (Ambion) with Lipofectamine RNAi Max (Invitrogen) following manufacturer's instructions and incubated for $30 \mathrm{~h}$ before performing ISH on coverslips or preparing RNA extracts. RNA extracts were prepared using the mirVana miRNA Isolation Kit (Ambion) following the manufacturer's instructions. In performing ISH on coverslips, cells were washed twice with PBS, fixed for $10 \mathrm{~min}$ with $1 \%$ formaldehyde (Thermo Scientific) in PBS for $10 \mathrm{~min}$, washed with PBS, permeabilized with $0.2 \%$ Triton X-100 (American Bioanalytical) for 20 min on ice, washed with PBS on ice, refixed with $4 \%$ formaldehyde in PBS for $10 \mathrm{~min}$ at room temperature, then peroxidase blocked with $3 \% \mathrm{H}_{2} \mathrm{O}_{2}$ for $10 \mathrm{~min}$. Coverslips were then prehybridized at $40^{\circ} \mathrm{C}$ for $30 \mathrm{~min}$, hybridized with double DIG $100 \mathrm{nM}$ miR-205 or scrambled probe, or $50 \mathrm{nM} \mathrm{U6}$ probe $5^{\prime}$ DIG labeled (Exiqon) for $1 \mathrm{~h}$, washed with $2 \times$ SSC at hybridization temperature twice then once at room temperature, blocked with $2 \%$ BSAPBS for $30 \mathrm{~min}$, then incubated with sheep anti-DIG-POD, Fab fragments from sheep (Roche Diagnostics) diluted 1:100 in block for $1 \mathrm{~h}$. The miRNA signal is detected with the TSA Cyanine five systems (Perkin Elmer) and the coverslips were mounted with Prolong Gold-DAPI (Molecular Probes).

\section{MiRNA qRT-PCR}

RNA concentrations were determined by the NanoDrop 2000 (Thermo Scientific), then reverse transcribed using the TaqMan microRNA Reverse Transcription Kit (Applied Biosystems, Foster City, CA, USA), and real-time PCR using the TaqMan microRNA assay kit for miR-205 or RNU6B (Applied Biosystems). RT-PCR was performed using the CFX96 machine (BioRad, Hercules, CA, USA). Reactions were done in triplicate along with no template control reactions.

\section{Statistical Analysis}

All values shown are mean \pm s.d. unless otherwise stated. Box plots show standard box and whisker plots where the error bars represent the $90^{\text {th }}$ and $10^{\text {th }}$ percentiles. Survival curves were generated by Kaplan-Meier analysis and tested for significance using the Mantel-Cox log-rank test. The prognostic significance for miR-205 was also determined using the Cox proportional hazards model. To test for differences between groups $P$-values were calculated by ANOVA analysis. All statistical analysis was done using Statview 5.0 (SAS Institute).

\section{RESULTS}

miR-205 has been independently shown to act as a tumor suppressor in melanoma where its expression was decreased in melanoma specimens compared to nevi. ${ }^{6,9,10}$ To confirm this observation using in situ methods and to determine its prognostic value, we validate a method of qISH in which the miR-205 ISH is multiplexed with S100/GP100 and DAPI in order to apply the AQUA measurement technology. This co-localization based approach uses the S100/GP100 signal to establish a tumor mask and allows for the measurement of miR-205 expression within the tumor mask resulting in an AQUA score directly proportional to the number of molecules per unit of area within melanoma cells. ${ }^{15}$ In evaluating nearly 600 melanoma specimens there was a large dynamic range in signal intensity, and expression was exclusively cytoplasmic in the majority of primary and metastatic melanoma cases (Figure 1). There was also substantial stromal expression in some cases, but stromal expression is excluded from measurement by the masking using S100/GP100. The U6 positive control probe revealed strong nuclear staining, and the scrambled negative control 

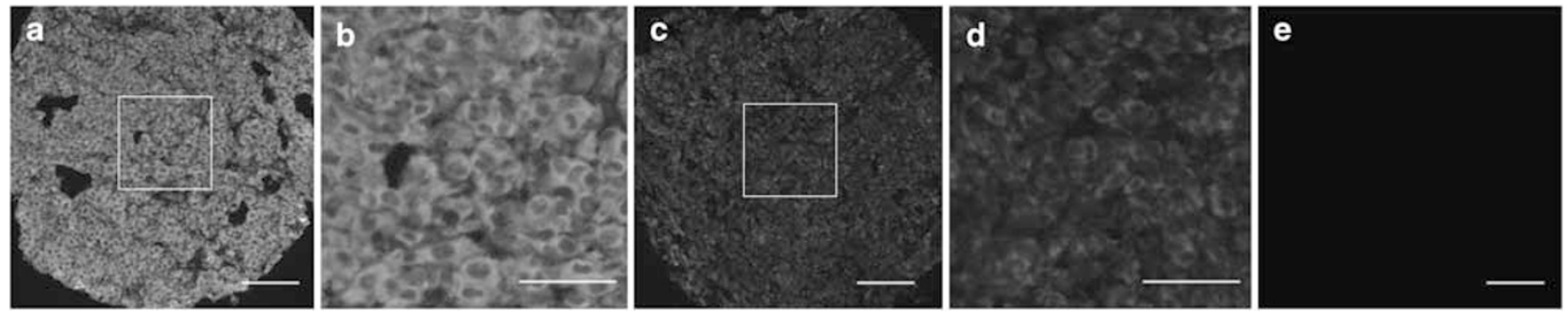

Figure 1 (a) Example of a primary melanoma specimen with high miR-205 expression. (b) High magnification of inset indicated in (a). (c) Primary melanoma specimen with low miR-205 expression. (d) High magnification of inset indicated in (c). (e) Example of scrambled probe negative control in serial section of patient tissue from (a). Scale bars represent $50 \mu \mathrm{m}$ in (a, c, e) and $25 \mu \mathrm{m}$ in (b), (d).
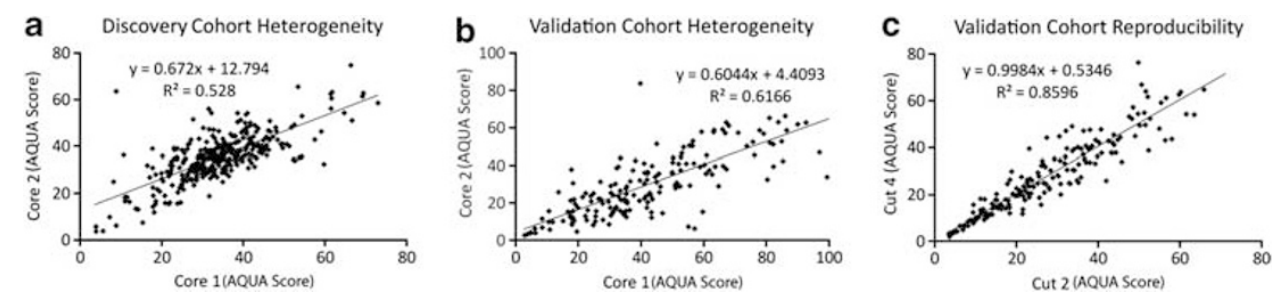

Figure 2 Reproducibility of miR-205 qISH as performed on redundant builds (different cores) of the (a), discovery cohort and the (b), validation cohort. (c) Reproducibility of miR-205 qISH as performed on serial sections of the validation cohort.

probe resulted in no signal (Figure 1 and data not shown). To determine reproducibility, the assay was performed on redundant cores of the discovery and validation cohort with $R^{2}>0.5$ and serial sections of the validation cohort with an $R^{2}=0.86$ representing acceptable reproducibility considering heterogeneity of different cores (Figure 2).

Specificity of the assay was first established using a miR205 specific unlabeled blocking oligo as well as a mutated blocking oligo with three nucleotide changes to compete for hybridization with the miR-205 probe. As expected hybridization in the presence of the specific blocking oligo resulted in no detectable signal and in the presence of the mutated oligo did not significantly alter the signal (figure not shown). Specificity was further evaluated using Mel-501 cell lines transfected with miR-205 mimic because Mel-501 cells express low levels of miR-205. In addition, A431 cells were transfected with miR-205 inhibitor (Figure 3) as A431 cells express high levels of miR-205. The concomitant increase or decrease in expression was then assessed using qISH and qRT-PCR as illustrated and quantified in Figure 3a-d. Although variability was detected with each method, there was a similar increase in expression as measured by both techniques in Mel-501 cells transfected with the miR-205 mimic and a similar decrease in expression in A431 cells transfected with miR-205 inhibitor.

In order to evaluate the prognostic value of miR-205 we first used the Yale Melanoma Discovery Cohort TMA. Similarly to Dar et $a l^{6}$ we found that miR-205 expression was decreased in metastatic and primary melanomas compared to nevi (Figure $4 \mathrm{a}$ ). The 110 primary melanomas with sufficient tumor area for analysis and complete follow-up data were divided into tertiles based on expression of miR-205. The top two tertiles were overlapping so they were combined into a single group. The lowest tertile was compared with the upper two tertiles revealing a significantly shorter disease-specific survival for the lowest expressing group (Figure $4 \mathrm{~b}$ ). This cut-point between high and low expression was also significant in univariate and multivariate analysis (Table 2) independent of age, sex, stage, and Breslow depth $(n=105$ with all clinicopathological variables).

The same AQUA score cut-point between high and low expression was then applied to the validation cohort consisting of 206 evaluable primary melanoma specimens with follow-up data (after median normalizing the scores on both cohorts for translation of the AQUA score). Again the low expressing group had a statistically significant shorter disease-specific survival (Figure 4c) that is also significant by univariate and multivariate analysis (Table 2). Interestingly when the primary melanoma patients are stratified according to miR-205 status and Breslow thickness, patients with Breslow $>2 \mathrm{~mm}$ and low miR-205 expression had a significantly shorter melanoma-specific survival compared with the other patients including those with thick Breslow and high miR-205 (Figure 5).

\section{DISCUSSION}

Although several studies have demonstrated the utility of miRNA ISH in FFPE specimens using DIG labeled LNAmodified probes, ${ }^{14,16,17}$ most studies have been descriptive, semi-quantitative, or performed on small cohorts. Here, we show the utility of investigating miRNAs as tissue biomarkers in large patient TMA cohorts using quantitative ISH. This method allows the visualization and measurement of miRNA expression in individual cells unlike most miRNA detection 

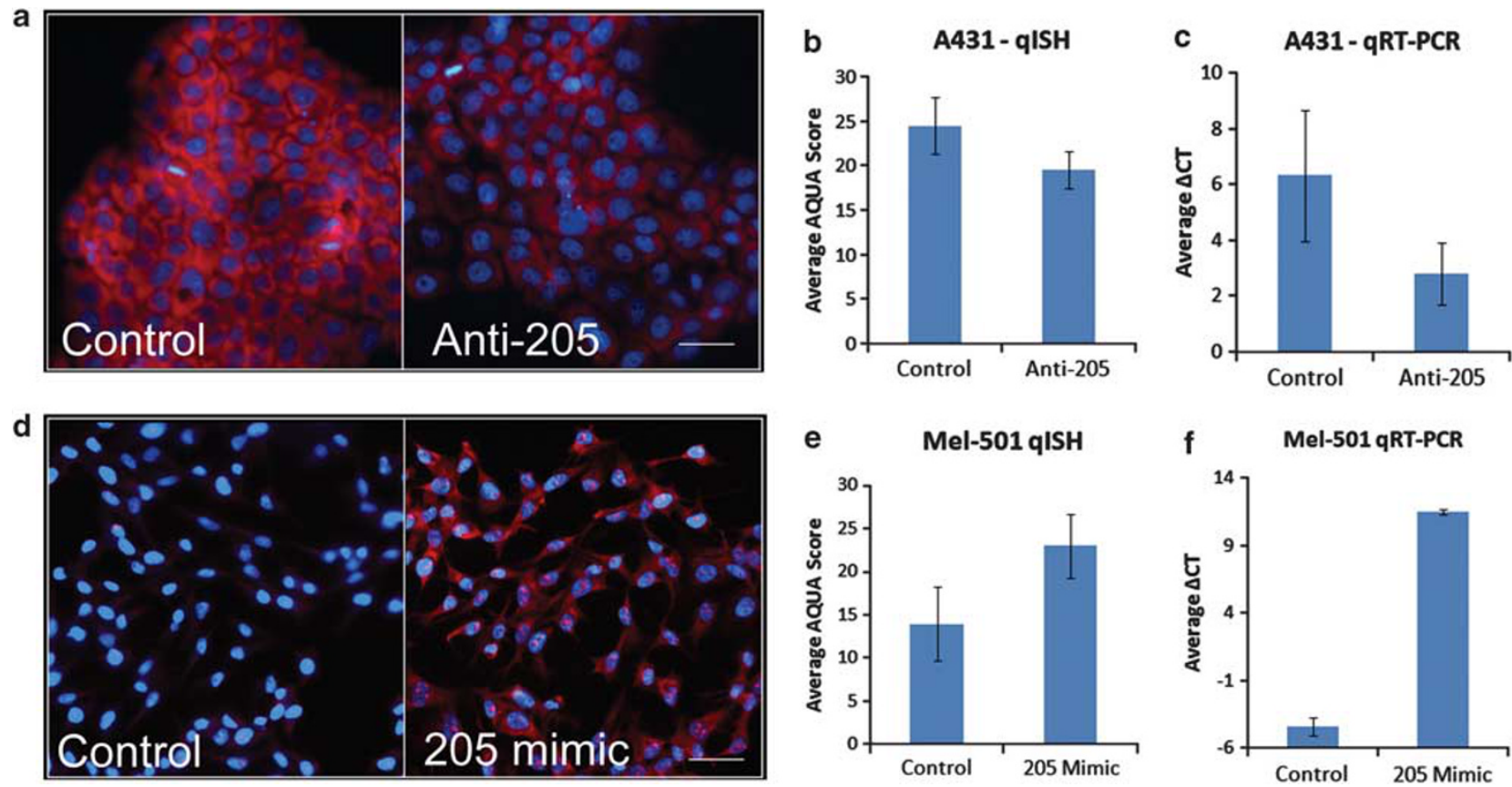

Figure 3 (a) Representative example of miR-205 ISH (red) performed on A431 cells transfected with the negative control miRNA inhibitor or miR-205 miRNA inhibitor merged with DAPI (blue). (b) Quantification of miR-205 knockdown by qISH from 24 random fields. (c) Quantification of miR-205 knockdown by qRT-PCR normalized by U6. (d) Representative example of miR-205 ISH performed on Mel-501 cells transfected with the mimic negative control or miR-205 mimic. (e) Quantification of miR-205 overexpression by qISH from 24 random fields. (f) Quantification of miR-205 overexpression by qRT-PCR normalized by U6. Scale bars represent $25 \mu \mathrm{m}$.
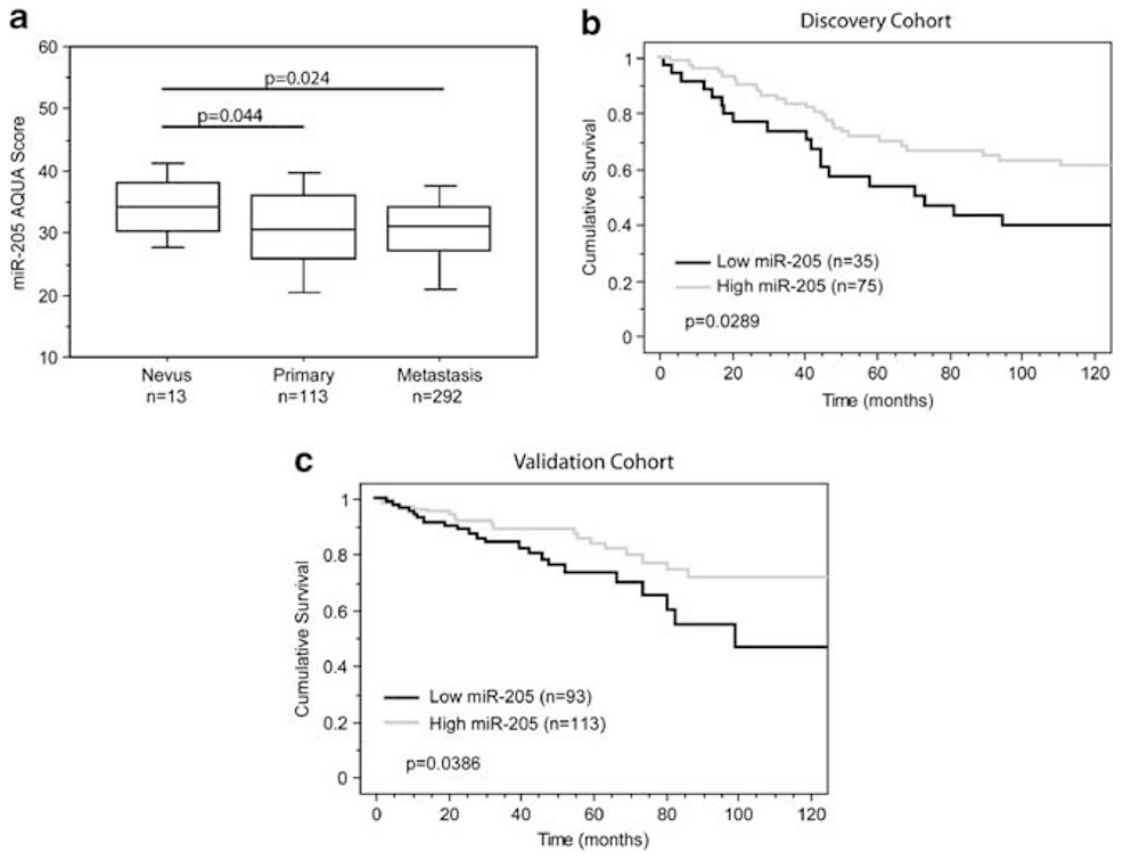

Figure 4 (a) Box plot demonstrating the distribution of miR-205 expression in nevi, primary, and metastatic melanoma specimens. (b) Kaplan-Meier survival analysis of miR-205 expression on the discovery cohort ( $P$-value calculated by the log-rank test). (a) Kaplan-Meier survival analysis of miR-205 expression on the Validation Cohort using the same cutoff score between high and low expression as the Discovery Cohort.

methods, which require total RNA extracts and lack critical spatial information. This spatial information can be important to understanding the biological function of cancer biomarkers. As illustrated in a study by Sempere et $\mathrm{al}^{17}$, deregulation of miR-126 and miR-155 as suggested in several cancer types may be confined to endothelial and 
Table 2 Univariate and multivariate analysis for 10-year melanoma-specific mortality

\begin{tabular}{|c|c|c|c|c|c|c|}
\hline & \multicolumn{3}{|c|}{ Univariate analysis } & \multicolumn{3}{|c|}{ Multivariate analysis } \\
\hline & $\mathrm{HR}$ & $95 \% \mathrm{Cl}$ & $P$-value & $\mathrm{HR}$ & $95 \% \mathrm{Cl}$ & $P$-value \\
\hline \multicolumn{7}{|l|}{$n=105$} \\
\hline \multicolumn{7}{|c|}{ Discovery cohort } \\
\hline \multicolumn{7}{|l|}{ Breslow } \\
\hline$<2.0 \mathrm{~mm}$ & 1 & & & 1 & & \\
\hline$>2.0 \mathrm{~mm}$ & 1.931 & $1.003-3.721$ & 0.0491 & 1.828 & $0.922-3.626$ & 0.0843 \\
\hline Age, years & 1.019 & $0.997-1.041$ & 0.0917 & 1.022 & $0.997-1.047$ & 0.0796 \\
\hline \multicolumn{7}{|l|}{ Sex } \\
\hline Male & 1 & & & 1 & & \\
\hline Female & 0.907 & $0.495-1.662$ & 0.752 & 1.085 & $0.575-2.047$ & 0.802 \\
\hline \multicolumn{7}{|c|}{ Stage at diagnosis } \\
\hline Localized & 1 & & & 1 & & \\
\hline Regional & 2.596 & $1.062-6.157$ & 0.0363 & 2.561 & $1.045-6.299$ & 0.0398 \\
\hline Distant & 3.205 & $1.242-8.268$ & 0.016 & 4.169 & $1.574-11.039$ & 0.0041 \\
\hline \multicolumn{7}{|c|}{ miR-205 expression } \\
\hline Low & 1 & & & 1 & & \\
\hline High & 0.534 & $0.288-0.990$ & 0.0463 & 0.4 & $0.207-0.773$ & 0.0064 \\
\hline \multicolumn{7}{|l|}{$n=192$} \\
\hline \multicolumn{7}{|c|}{ Validation cohort } \\
\hline \multicolumn{7}{|l|}{ Breslow } \\
\hline$<2.0 \mathrm{~mm}$ & 1 & & & 1 & & \\
\hline$>2.0 \mathrm{~mm}$ & 3.014 & $1.489-6.101$ & 0.002 & 2.603 & $1.186-5.714$ & 0.0171 \\
\hline Age, years & 1.022 & $1.000-1.045$ & 0.052 & 1.031 & $1.005-1.057$ & 0.0169 \\
\hline \multicolumn{7}{|l|}{ Sex } \\
\hline Male & 1 & & & 1 & & \\
\hline Female & 0.53 & $0.262-1.069$ & 0.076 & 0.557 & $0.263-1.177$ & 0.125 \\
\hline \multicolumn{7}{|c|}{ Stage at diagnosis } \\
\hline Localized & 1 & & & 1 & & \\
\hline Regional & 2.686 & $1.316-5.484$ & 0.0066 & 2.189 & $1.017-4.713$ & 0.0452 \\
\hline Distant & 7.726 & $1.80-33.10$ & 0.0059 & 28.238 & $5.52-144.42$ & $<0.001$ \\
\hline \multicolumn{7}{|c|}{ miR-205 expression } \\
\hline Low & 1 & & & 1 & & \\
\hline High & 0.506 & $0.266-0.965$ & 0.0386 & 0.511 & $0.262-0.997$ & 0.0489 \\
\hline
\end{tabular}

Abbreviations: $\mathrm{Cl}$, confidence interval; $\mathrm{HR}$, hazard ratio. 

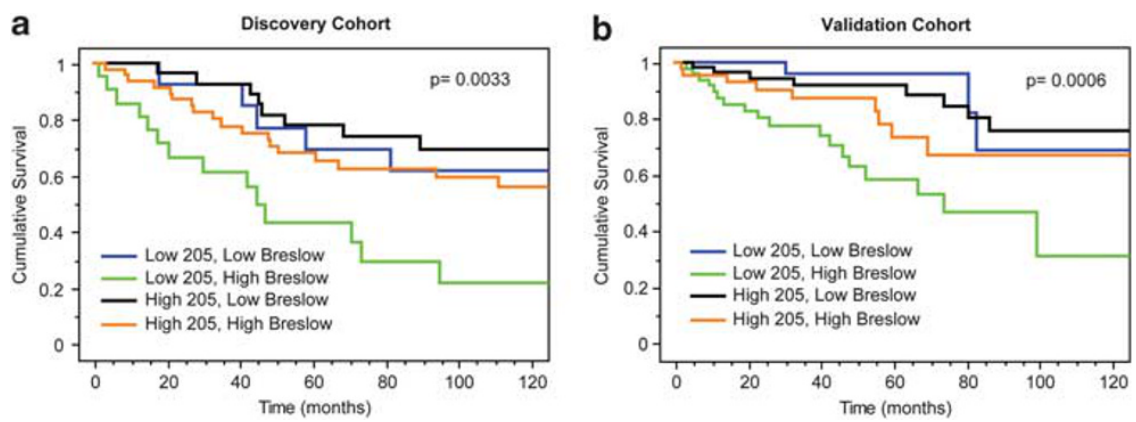

Figure 5 (a) Kaplan-Meier survival analysis of patients stratified by miR-205 and Breslow thickness where high Breslow is $>2.0 \mathrm{~mm}$ and low Breslow is $<2.0 \mathrm{~mm}$ on the discovery cohort (low 205/low Breslow $n=14$, low 205/high Breslow $n=21$, high 205/low Breslow $n=27$, high 205/high Breslow $n=48$ ). (b) Kaplan-Meier survival analysis as in (a), but on the validation cohort (low 205/low Breslow $n=33$, low 205/high Breslow $n=54$, high 205/ low Breslow $n=58$, high 205/high Breslow $n=47$ ). $P$-value calculated by the log-rank test.

immune cells, respectively, and not the malignant tumor cells as previously suggested using RT-PCR and microarray studies. miR-205 has been shown to be expressed in the stroma of mouse mammary tissue and we found stromal expression as well. ${ }^{18}$ However, the AQUA technology allows the measurement of expression in the malignant melanocytic cells as determined by coexpression with S100/GP100, thus validating the contribution of miR-205 specifically in the malignant cells. The loss or significant decrease in expression in the most aggressive tumors is supportive of the role of miR-205 as a tumor suppressor miRNA.

As with any new technology, the assay must be validated to prove reproducibility and specificity. In this study, we validated reproducibility on serial sections and redundant cores with $R^{2}=0.8$ and $>0.5$, respectively. In addition, serial sections of control index arrays are ran alongside as quality control measures (data not shown). Specificity of the assay is supported by specific and mutated blocking oligos and cell lines transfected with a miR-205 mimic and a miR-205 inhibitor.

Although the role of miR-205 in cancer is controversial, it has been shown to be upregulated in lung, bladder, and ovarian cancer, ${ }^{19-21}$ whereas downregulated in breast, prostate, and melanoma $a^{6,8,9,22}$ suggesting a tissue type dependent role in cancer. However, the miR-205 targets include such oncogenes as ErbB3, E2F1, VEGFA, and $\mathrm{PKC} \varepsilon, 6,8,23,24$ which support its role as a tumor suppressor. It is also involved in regulating the epithelial to mesenchymal transition by downregulating Zeb1. ${ }^{7}$ In melanoma, miR-205 has been shown to be downregulated in primary and metastatic melanoma compared with nevi by qRT-PCR and microarray analysis. ${ }^{6,9,10}$ Here, we confirm those observations using a third method. Although the difference seen here between nevi, primary and metastatic melanoma specimens as measured by qISH appears less than that detected by qRTPCR, we believe this is owing to a decreased dynamic range of qISH compared with qRT-PCR. Furthermore, when overexpressed in melanoma cell lines, miR-205 suppressed tumor growth in vivo, induced senescence, and reduced the proliferative capacity of cells by means of downregulating E2F1. Overall, we believe that the bulk of the evidence supports a tumor suppressor role for miR-205.

Finally, we show the prognostic value of miR-205 in two independent cohorts totaling over 300 primary melanoma specimens. In addition, miR-205 expression level was able to stratify patients with high Breslow thickness into high and low risk groups, however, in our cohorts was unable to stratify patients of low Breslow thickness likely because of the small number of patients. Although this result represents an exploratory study on two retrospective cohorts, it suggests potential value for stratification of melanoma outcome. As other miRNAs are examined, it is possible that they may be valuable for inclusion in melanoma diagnostic tests that predict recurrence or response to therapy in melanoma.

\section{ACKNOWLEDGEMENTS}

Thanks to Emily Storch and Lori Charette of the Yale Pathology Tissue Services TMA facility for collection of tissue and construction of the TMAs. This work was funded by R01 CA 114277 to DLR.

\section{DISCLOSURE/CONFLICT OF INTEREST}

Dr Rimm is a co-founder, consultant for and stockholder in HistoRx, the exclusive licensee of the Yale-owned AQUA technology.

1. Croce CM.. Causes and consequences of microRNA dysregulation in cancer. Nat Rev Genet 2009;10:704-14.

2. Friedman $\mathrm{RC}$, Farh $\mathrm{KK}$, Burge $\mathrm{CB}$, et al. Most mammalian mRNAs are conserved targets of microRNAs. Genome Res 2009;19:92-105.

3. Lewis BP, Burge CB, Bartel DP. Conserved seed pairing, often flanked by adenosines, indicates that thousands of human genes are microRNA targets. Cell 2005;120:15-20.

4. Lim LP, Lau NC, Weinstein EG, et al. The microRNAs of Caenorhabditis elegans. Genes Dev 2003;17:991-1008.

5. Kasinski AL, Slack FJ. Epigenetics and genetics. MicroRNAs en route to the clinic: progress in validating and targeting microRNAs for cancer therapy. Nat Rev Cancer 2011;11:849-64.

6. Dar AA, Majid S, de Semir D, et al. miRNA-205 suppresses melanoma cell proliferation and induces senescence via regulation of E2F1 protein. J Biol Chem 2011;286:16606-14.

7. Gregory PA, Bert AG, Paterson EL, et al. The miR-200 family and miR205 regulate epithelial to mesenchymal transition by targeting ZEB1 and SIP1. Nat Cell Biol 2008;10:593-601. 
8. Wu H, Zhu S, Mo YY.. Suppression of cell growth and invasion by miR205 in breast cancer. Cell Res 2009;19:439-48.

9. Philippidou D, Schmitt M, Moser D, et al. Signatures of microRNAs and selected microRNA target genes in human melanoma. Cancer Res 2010;70:4163-73.

10. $\mathrm{Xu} \mathrm{Y,Brenn} \mathrm{T,} \mathrm{Brown} \mathrm{ER,} \mathrm{et} \mathrm{al.} \mathrm{Differential} \mathrm{expression} \mathrm{of} \mathrm{microRNAs}$ during melanoma progression: miR-200c, miR-205 and miR-211 are downregulated in melanoma and act as tumour suppressors. $\mathrm{Br} J$ Cancer 2012;106:553-561.

11. Bishop JA, Benjamin $\mathrm{H}$, Cholakh $\mathrm{H}$, et al. Accurate classification of nonsmall cell lung carcinoma using a novel microRNA-based approach. Clin Cancer Res 2010;16:610-9.

12. Gould Rothberg BE, Berger AJ, Molinaro AM, et al. Melanoma prognostic model using tissue microarrays and genetic algorithms J Clin oncol 2009;27:5772-80.

13. Hanna JA, Wimberly $\mathrm{H}$, Kumar $\mathrm{S}$, et al. Quantitative analysis of microRNAs in tissue microarrays by in situ hybridization. BioTechniques 2012;52:235-45.

14. Pena JT, Sohn-Lee $\mathrm{C}$, Rouhanifard $\mathrm{SH}$, et al. miRNA in situ hybridization in formaldehyde and EDC-fixed tissues. Nat Methods 2009; 6:139-41.

15. Camp RL, Chung GG, Rimm DL. Automated subcellular localization and quantification of protein expression in tissue microarrays. Nat Med 2002;8:1323-7.
16. Nuovo GJ. In situ detection of microRNAs in paraffin embedded, formalin fixed tissues and the co-localization of their putative targets. Methods 2010;52:307-15.

17. Sempere LF, Preis M, Yezefski T, et al. Fluorescence-based codetection with protein markers reveals distinct cellular compartments for altered MicroRNA expression in solid tumors. Clin Cancer Res 2010;16:4246-55.

18. Avril-Sassen S, Goldstein LD, Stingl J, et al. Characterisation of microRNA expression in post-natal mouse mammary gland development. BMC Genomics 2009;10:548.

19. Gottardo F, Liu CG, Ferracin M, et al. Micro-RNA profiling in kidney and bladder cancers. Urologic Oncol 2007;25:387-92.

20. Iorio MV, Visone R, Di Leva G, et al. MicroRNA signatures in human ovarian cancer. Cancer Res 2007;67:8699-707.

21. Yanaihara N, Caplen N, Bowman E, et al. Unique microRNA molecular profiles in lung cancer diagnosis and prognosis. Cancer Cell 2006;9:189-98.

22. Majid S, Dar AA, Saini S, et al. MicroRNA-205-directed transcriptional activation of tumor suppressor genes in prostate cancer. Cancer 2010;116:5637-49.

23. Gandellini P, Folini $M$, Longoni $N$, et al. miR-205 Exerts tumorsuppressive functions in human prostate through down-regulation of protein kinase Cepsilon. Cancer Res 2009;69:2287-95.

24. Iorio MV, Casalini P, Piovan C, et al. microRNA-205 regulates HER3 in human breast cancer. Cancer Res 2009;69:2195-200. 